\title{
Zero Spatial Frequency Limit: Method to Characterize Photopolymers as Optical Recording Material
}

\author{
Sergi Gallego, ${ }^{1,2}$ Andrés Márquez, ${ }^{1,2}$ Manuel Ortuño, $, 1,2$ \\ Cristian Neipp, ${ }^{1,2}$ Inmaculada Pascual, ${ }^{2,3}$ and Augusto Beléndez ${ }^{1,2}$ \\ ${ }^{1}$ Departamento de Física, Ingeniería de Sistemas y Teoría de la Señal, Universidad de Alicante, Apartado 99, 03080 Alicante, Spain \\ ${ }^{2}$ Instituto Universitario de Física Aplicada a las Ciencias y las Tecnologías, Universidad de Alicante, Apartado 99, \\ 03080 Alicante, Spain \\ ${ }^{3}$ Departamento de Óptica, Farmacología y Anatomía, Universidad de Alicante, Apartado 99, 03080 Alicante, Spain
}

Correspondence should be addressed to Sergi Gallego, sergigir@gmail.com

Received 3 May 2012; Accepted 14 June 2012

Academic Editor: Michael R. Gleeson

Copyright ( 2012 Sergi Gallego et al. This is an open access article distributed under the Creative Commons Attribution License, which permits unrestricted use, distribution, and reproduction in any medium, provided the original work is properly cited.

\begin{abstract}
Photopolymers are useful for different holographic applications such as holographic data storage or diffractive optical elements. However, due to the presence of two different phenomena, polymer formation and monomer diffusion, it is difficult to characterize each parameter independently. We propose a direct method based on zero spatial frequency recording, to eliminate the diffusion influence, and on interferometric techniques, both in transmission and in reflection, to obtain quantitative values of shrinkage, polymerization rate, polymer refractive index and relation between intensity and polymerization, and so forth, This method has been implemented in the Holography and Optical Processing Group from the University of Alicante to characterize different photopolymers. In this paper, we present a compilation of the results obtained with this method for different photopolymers and we compare their characteristics.
\end{abstract}

\section{Introduction}

Photopolymers are useful for different applications due to the refractive index variations and relief profiles generated $[1-8]$. There are many types of photopolymers that may be differentiated by the type of binder, since this component determines to a great extent the choice of monomer, dye, and initiator used in the photopolymer. Normally, these materials are used in holographic applications, where high values of spatial frequencies are recorded. In these range of frequencies many processes are involved in the hologram formation, such as species diffusion, nonlocal polymerization (due to the finite size of polymer chains), shrinkage or swelling (volume changes), and others [9-13]. Therefore, analyzing the behaviour of a photopolymer as an optical storage medium is a complicate task. In this sense, the variations in the estimation of the monomer diffusion affect significantly the values obtained for other material parameters, making difficult to calculate them separately. In particular, it is interesting to analyze the material behavior at very low spatial frequencies, since at these frequencies monomer diffusion does not play an important role [14] and even disappears at the zero spatial frequency limit [15-17].

Zero spatial frequency limit analysis is a method is based on an interferometer that has been successfully applied in the phase-shift versus applied voltage characterization of liquidcrystal displays (LCDs) [18]. In the limit of zero frequency recording, that is, uniform exposure of the photosensitive layer, no diffusion processes are produced [15-17] and we can determined directly many parameters (as opposed to holographic characterization, the data obtained in this case are directly related to the physical properties of the material without the need of nonlinear fitting of multiparametric models) and these values can be used in diffusion models to predict the exact holographic material behavior $[12,13]$. The measuring of refractive index variation and the changes in the thickness, in noncover-plated samples only (where the light is reflected directly from the material), can be directly observed in real time. Some other parameters that can be measured are polymerization rate, polymer refractive index 
and relation between polymerization and recording intensity, and so forth. Interferometric measurements are applied to calculate the phase shift between transmitted and reflected beams. Measuring the phase variation of the reflected beam on the zone where polymerization takes place, we obtain direct information about the changes in the material thickness, and analyzing the phase changes in the transmitted beam in the same area we obtain information about changes in the refractive index and thickness together. The interferometric method proposed has been applied with success to characterize some materials. In this work we present a review and new comparison between different photopolymers. In this paper we present a compilation of the results obtaining with this method for different photopolymers presented in the following references [15-17] with some new experiments and we compare their characteristics. At first we present the results obtained for some materials with polyvinyl alcohol (PVA) as a binder. One of them has Acrylamide (AA) as a main monomer. This photopolymer have been studied for many groups in many different cases [19-23]. Nevertheless, $\mathrm{AA}$ has an undesirable feature, the toxicity of AA and its low environmental compatibility $[24,25]$. Therefore, we have changed the AA by sodium acrylate, NaAO, in order to obtain a higher environmental compatibility. Then we present the study of the recovered this material at the zero spatial frequency limit. The shrinkage of recovered materials cannot be measured which is an important drawback in the characterization of this material with zero spatial frequency recording. Finally, we present how the zero spatial frequency recording can be applied successfully also to H-PDLC materials.

\section{Materials and Experimental Setup}

The zero spatial frequency method (Figures $1(\mathrm{a})$ and $1(\mathrm{~b})$ ) is based on an interferometer that has been successfully applied in the phase-shift versus applied voltage characterization of liquid-crystal displays (LCDs) [18]. It shows good precision, and, due to its quasi-common-path architecture, is a robust setup, less sensitive to changing environmental conditions, and simpler to construct than Mach-Zehnder type interferometers. The setup has two arms with an angular separation of $14^{\circ}$, one to expose the recording material, whereas the second arm is the interferometer used to measure, in real time, the phase-shift. The recording material is perpendicularly oriented with respect to the interferometer axis in order to simplify the analysis of the interferometric results: at an oblique incidence we should take into account both the Fresnel coefficients at the interface and the increase of distance in the propagation across the layer.

In the first arm, the exposure beam provided by a solidstate Nd-YVO4 Verdi laser with a wavelength of $532 \mathrm{~nm}$ (at this wavelength the dye presents the maximum absorption) is expanded and collimated using a spatial filter and a lens, obtaining a beam with $1 \mathrm{~cm}$ of radius. A wave plate and a neutral filter (attenuator) are added before the spatial filter to control the orientation and the intensity of the linearly polarized beam produced by the laser Nd-YVO4. A polarizer
(P), with its transmission axis oriented along the vertical of the lab, is introduced to produce a beam with TE polarization incident onto the recording material. This incident beam forms an angle of $14^{\circ}$ with respect to the photopolymer layer.

In the second arm we use a He-Ne laser to generate the interferences pattern, since the photopolymer does not present any absorption at $633 \mathrm{~nm}$. We introduce a grating with a spatial frequency of 4 lines/mm to generate a series of diffracted orders; we block all the orders except +1 and -1 . One of the two orders impinges on the exposed zone (illuminated by the Nd-YVO4 laser, $532 \mathrm{~nm}$ ) and the other one impinges on the nonexposed zone. The distance between the two orders is more than $1 \mathrm{~cm}$, so as to eliminate the influence of the monomer diffusion in the polymerization process. Afterwards the orders are brought to interfere and from the interference pattern we may obtain the evolution in the material due to polymerization (diffusion is not present).

In Figure 1(a), once the two orders have propagated throughout the photopolymer, a lens is used to make them interfere. A microscope objective is used to amplify the interference pattern onto a CCD camera. This pattern is captured in real time as a function of exposure at specific time intervals, then is digitized and transferred to a personal computer [15]. We note that there may appear reflection gratings generated by Fresnel boundary reflections. However the recording of these nondesired reflection gratings probably is highly attenuated due to the following reasons: the last boundary reflection between glass and air is weak and in addition, due to the nonlocal effect, the size of polymer chains prevents the recording of these high spatial frequencies.

In Figure 1(b) once the two orders are reflected by the photopolymer, we place a lens to make them interfere. A microscope objective is used to amplify the interference pattern onto a CCD camera. This pattern is captured at specific exposure time intervals, and then it is digitized and transferred to a personal computer. The reflections from the back surface are eliminated using a black film on the back surface [16].

Once the interference pattern has been stored, we can measure the shift with respect to the initial pattern obtained for the unexposed layer. To increase the accuracy in this calculation, we cross-correlate the different interference patterns with the initial one. The cross-correlation produces a clear peak [15-17]. The location of this peak for each correlated interference pattern with respect to the center of the image is equal to the shift in the fringe pattern. A full fringe shift is equal to a $2 \pi$ radians variation in the phase shift. In this experiment, as we are using a reflection interferometer, the phase shift is directly related to the thickness variation of the material [16]. Depending on the sense of movement of the fringes (indicated by the arrow in the Figure 1) we can deduce if the exposed zone is shrinking or swelling with respect to the nonexposed one. In the case of the photopolymer studied in this paper we obtained that the exposed zone shrinks with respect to the nonexposed area.

When PVA is used as a binder the monomer must be water soluble, it must not evaporate during the preparation of layers (drying process) and it has to react through 


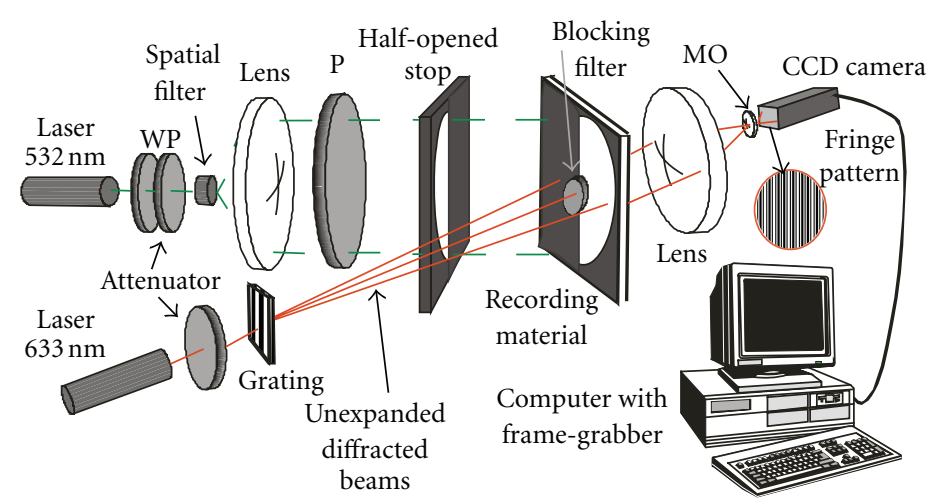

(a)

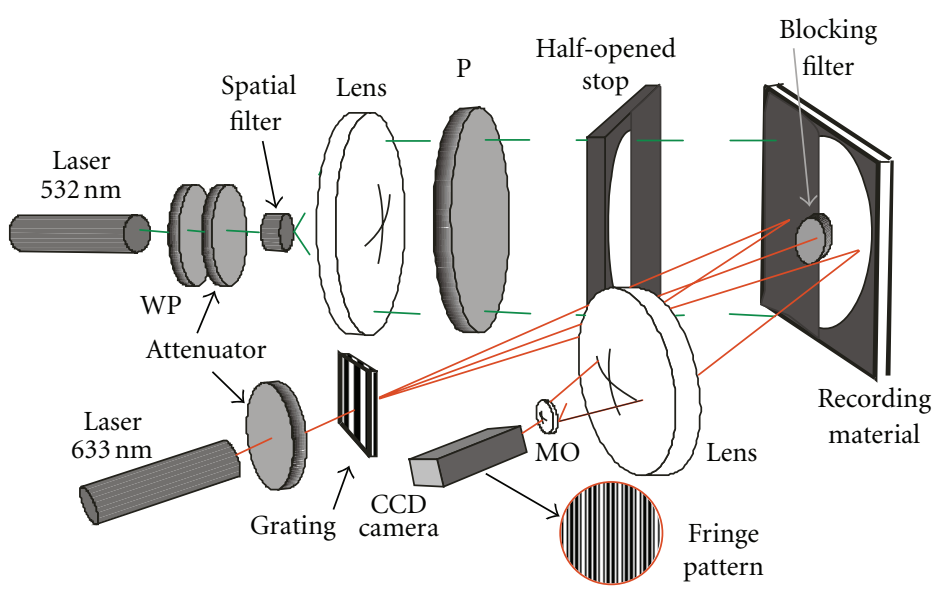

(b)

FIGURE 1: Experimental setup for zero spatial frequency analysis. P is polarizer, WP is wave plate, MO is microscope objective. (a) Transmission analysis. (b) Reflection analysis.

a radical chain mechanism. As mentioned above the toxicity of sodium acrylate $(\mathrm{NaAO})$ is lower than that of AA [17]. Moreover, different triethanolamine (TEA) concentrations are normally used to obtain different material properties (TEA is a liquid at ambient temperature and plays an important role in the monomer and polymer diffusion during the polymerization process too).

The less toxic component of PVA/AA standard photopolymer is the dye, yellowish Eosin (YE), nevertheless to obtain a real environmental compatibility we have tested a less toxic dye $[23,24]$. In the new photopolymer we use the sodium salt $5^{\prime}$-riboflavin monophosphate (PRF) as dye, bearing in mind that this substance is water soluble and exists in the environment, so it is not likely to cause environmental problems.

The solutions, whose compositions can be seen in Table 1, with water as solvent, and PVA as binder $(\mathrm{Mw}=$ 130000 a.m.u., hydrolysis degree $=87.7 \%$ ) are deposited, using the force of gravity over a glass. The glass surface measures $30 \times 40 \mathrm{~cm}^{2}$ and left inside a dark chamber where humidity and temperature are controlled (relative humidity $\left.=40-45 \%, \mathrm{~T}=21-23^{\circ} \mathrm{C}\right)$ [17]. When large part of the water has evaporated (about 48 hours), the layer has enough mechanical resistance and it can be cut without deformation. As crosslinker we have used N, N'-methylenebis-acrylamide (BMA) to study the mixture with NaAO. The presence of BMA in the formulation of biophotopol [25] is optional and not essential to the main features of the photopolymer. Furthermore, the toxicity of BMA is clearly lower than AA. In this work we have characterized five new compositions. We have tested photopolymers with different TEA concentrations, with different concentrations of BMA, and different dyes. Composition A is directly derived from the previous works where biophotopol composition is optimized to achieve thick layers (around $1 \mathrm{~mm}$ thick). Since we have found weak modulation of layer type A, we have introduced BMA as crosslinker in compositions B and C. To increase the differences between exposed and nonexposed zones we have also introduced more $\mathrm{NaAO}$ in compositions $\mathrm{B}$ and D. To substitute YE we have presented composition E. The standard compositions used in previous works are compositions F and G (PVA/AA-based materials without and with crosslinker). The thickness of the samples is around $(100 \mu \mathrm{m})$ and the intensity at $532 \mathrm{~nm}$ used is $1 \mathrm{~mW} / \mathrm{cm}^{2}$. 
TABLE 1: Solution compositions and thicknesses.

\begin{tabular}{|c|c|c|c|c|c|c|c|}
\hline Composition type & $\mathrm{A}$ & $\mathrm{B}$ & $\mathrm{C}$ & $\mathrm{D}$ & E & $\mathrm{F}$ & G \\
\hline $\mathrm{NaAO}(\mathrm{g})$ & 0.75 & 1.5 & 0.75 & 1.5 & 0.75 & - & - \\
\hline $\mathrm{H}_{2} \mathrm{O}(\mathrm{mL})$ & 12.5 & 12.5 & 12.5 & 12.5 & 12.5 & 14.5 & 14.5 \\
\hline TEA $(\mathrm{mL})$ & 1.25 & 1.5 & 1.5 & 3 & 3 & 1.25 & 2.3 \\
\hline PVA $(\mathrm{mL})(15 \% \mathrm{w} / \mathrm{v})$ & 12.5 & 12.5 & 12.5 & 12.5 & 12.5 & 12.5 & 12.5 \\
\hline YE $(0.8 \% \mathrm{w} / \mathrm{v})(\mathrm{mL})$ & 0.6 & 0.6 & 0.6 & 0.6 & - & 0.6 & 0.6 \\
\hline PRF (g) & - & - & - & - & 0.14 & - & - \\
\hline $\mathrm{BMA}(\mathrm{g})$ & - & 0.1 & 0.2 & - & - & - & 0.25 \\
\hline $\mathrm{AA}(\mathrm{g})$ & - & - & - & - & - & 0.96 & 0.84 \\
\hline Thickness $\pm 4(\mu \mathrm{m})$ & 105 & 100 & 97 & 107 & 106 & 100 & 102 \\
\hline
\end{tabular}

TABLE 2: Chemical compositions of H-PDLCs layers analyzed.

\begin{tabular}{lccccc}
\hline & $\mathrm{C} 1$ & $\mathrm{C} 2$ & $\mathrm{C} 3$ & $\mathrm{C} 4$ & $\mathrm{C} 5$ \\
\hline DPHPA & $1.4 \mathrm{~g}$ & $1.5 \mathrm{~g}$ & $1.6 \mathrm{~g}$ & $1.8 \mathrm{~g}$ & $2 \mathrm{~g}$ \\
NMDETA & $0.4 \mathrm{~mL}$ & $0.4 \mathrm{~mL}$ & $0.4 \mathrm{~mL}$ & $0.4 \mathrm{~mL}$ & $0.4 \mathrm{~mL}$ \\
NVP & $0.1 \mathrm{~mL}$ & $0.1 \mathrm{~mL}$ & $0.1 \mathrm{~mL}$ & $0.1 \mathrm{~mL}$ & $0.1 \mathrm{~mL}$ \\
YEt & $1 \mathrm{mg}$ & $1 \mathrm{mg}$ & $1 \mathrm{mg}$ & $1 \mathrm{mg}$ & $1 \mathrm{mg}$ \\
LC BL087 & $0.6 \mathrm{~mL}$ & $0.5 \mathrm{~mL}$ & $0.4 \mathrm{~mL}$ & $0.2 \mathrm{~mL}$ & \\
\hline
\end{tabular}

In these materials the absence of cover plate will lead to two types of cross membrane diffusion taking place: oxygen across the boundary [26] and water in and/or out (due to heating or absorption).

The H-PDLC photopolymer is composed of dipentaerythritol penta/hexa-acrylate (DPHPA) as monomer and binder, $\mathrm{N}$-vinylpyrrolidone (NVP) as crosslinker, ethyl eosin (YEt) as dye, and N-methyl diethanolamine (NMDETA) as radical generator. These components were mixed under red light where the material is not sensitive and under a temperature of $40^{\circ} \mathrm{C}$ to solve properly all the components, specially the crystal liquid molecules (LC BL087 provided by Merck). The solution $(100 \mathrm{~mL})$ was sonicated in an ultrasonic bath, heated, mixed with a magnetic mixer, and deposited between two glass plates each $2 \mathrm{~mm}$ thick. The H-PDLCs layers have thickness around $12 \mu \mathrm{m}$ in order to apply the electric field and to align the crystal liquid molecules, thus obtain the switchable holographic gratings. In the samples analyzed in this work we have used spacing of $106 \mu \mathrm{m}$ to increase the phase shift and see properly the different behaviours reducing the error in our calculations [15]. We focus our attention on the characterization of the chemical composition; we do not make switchable gratings. In Table 2 we described the composition of different layers analyzed at zero spatial frequencies. These compositions are designed to keep the liquid volume constant. In the five compositions we analyzed the influence of the liquid crystal in the polymerization process.

In order to calculate an approximate value for the refractive index, we will assume that when exposition has finished, all the monomer has been polymerized. In this sense, it is important to obtain a precise estimation of the initial volume fraction of the monomer present in the layer [13, 27, 28]. This volume fraction it is more complicate to calculate in
TABle 3: Initial monomer volume fraction for PVA compositions analyzed.

\begin{tabular}{lcccccc}
\hline Composition type & A & B & C & D & F & G \\
\hline Initial monomer volume fraction & 0.13 & 0.15 & 0.25 & 0.17 & 0.20 & 0.22 \\
\hline
\end{tabular}

noncover-plated PVA materials, because PVA water absorption from the environment depends on the humidity. The water presence can be estimated if we measure the quantity of water evaporated during the drying process. It is interesting to note that after the drying process only very small portion of the initial water remains inside the layer. In our case, 48 hours after the layer deposition, the initial monomer volume fraction reaches the values presented in Table 3.

Once we have introduced the volume fractions, it is easy obtain the volume fraction for each component, $\phi^{(i)}$. Using the values of the refractive index, $n_{i}$, of each compound together with the Lorentz-Lorenz equation [27, 28], the refractive index of the layer before recording can be calculated, $n_{0}$ :

$$
\frac{n_{0}^{2}-1}{n_{0}^{2}+2}=\sum_{i=1}^{k} \frac{n_{i}^{2}-1}{n_{i}^{2}+2} \phi^{(i)}
$$

These values were checked using Abbe refractometer (Model AYA-15) measurements and are presented in Table 4. As can be deduced from Table 4, in compositions $\mathrm{C} 1$ and $\mathrm{C} 2$ the liquid crystal volume fraction is higher than $20 \%$. That can avoid huge variations in the refractive index at zero spatial frequency recording. $n_{0}$ is the average refractive index of the layer before the exposure. For zero spatial frequency analysis applied to H-PDLC, liquid crystal plays a similar role than binder in PVA materials; therefore, it does not participate in the chemical reactions.

\section{Theoretical Treatment of Experimental Data}

The phase shift in the reflection measurements, $\Delta \Phi_{\text {Shr }}$, is directly related to thickness variation during exposition at the zero spatial frequency limit. In this case, the thickness variations of the material $(\Delta d)$ can be expressed as follows:

$$
\Delta d=\frac{\Delta \Phi_{\mathrm{Shr}} \lambda \cos \alpha}{4 \pi},
$$


TABLE 4: Initial volume fractions and refractive indices, $n$.

\begin{tabular}{lcccccc}
\hline & C1 & C2 & C3 & C4 & C5 & $n$ \\
\hline DPHPA & 0.5241 & 0.5649 & 0.6061 & 0.6899 & 0.7758 & 1.4900 \\
NMDETA & 0.1729 & 0.1739 & 0.1750 & 0.1771 & 0.1792 & 1.4694 \\
NVP & 0.0433 & 0.0435 & 0.0437 & 0.0443 & 0.0448 & 1.5110 \\
YEt & 0.0002 & 0.0002 & 0.0002 & 0.0002 & 0.0002 & 1.7700 \\
LC BL087 & 0.2594 & 0.2175 & 0.1750 & 0.0885 & 0 & 1.5460 \\
$n_{0}$ & 1.5016 & 1.4993 & 1.4969 & 1.4922 & 1.4873 & \\
\hline
\end{tabular}

where $\lambda$ is the wavelength, $633 \mathrm{~nm}$ in our case, and $\alpha$ is the angle inside the material. At zero spatial frequency limit, when diffusion does not take place, the decreasing of the monomer concentration, $\phi^{(m)}$, depends on the recording intensity, $I$, the dependency of the polymerization on intensity, $\gamma$, the initial monomer concentration, and the polymerization reaction constant, $K_{R}[12]$ :

$$
\phi^{(m)}(t)=\phi_{0}^{(m)} e^{-I^{\gamma} K_{R} t},
$$

where $\phi^{(m)}$ is the initial monomer concentration in volume fraction.

The model used in this paper is simple and based on the classical model proposed by Zhao and Moroulis [12]. The aim of the paper is to propose and demonstrate a simple diffractive and interferometric method to calculate the first approximation of the polymerization rate and monomer diffusion. It is important to note that the gradient of monomer concentration in depth due to the dye absorption analyzed in previous works $[29,30]$ has been disregarded in this first approximation to the problem. In this sense it is important to mention the contributions of Sheridan et al. to clarify all the parameters involved in the polymerization process such as the influence of oxygen inhibiting [26], the effects of the dye kinetics, and polymer chain initiation, propagation, and termination [31-33] that can be studied using real zero analysis in future works. In any case the parameters obtained using the presented methodology produce good results when introduced in simple models as it is shown in references $[34,35]$. Following the same steps presented in [15-17] we can obtain the polymerization rate as a function of the experimental values of the phase shift during recording, PS, and the phase of saturation, $\mathrm{PS}_{\infty}$ :

$$
\ln \left(1-\frac{\mathrm{PS}(t)}{\mathrm{PS}_{\infty}}\right)=-I^{\gamma} K_{R} t=-F_{R} t
$$

Parameter, $\gamma$ (which governs the relation between recording intensity and polymerization rate, see (3)), can be associated to the viscosity of photopolymer system. In this sense values of $\gamma$ around 0.5 are associated to high fluid systems and for very dry systems values are near to $1[12,17]$. To calculate this parameter the material can be exposed with some different intensities.

Furthermore, if the quantities of different compounds are known, it is possible to obtain the polymer refractive index using Lorentz-Lorenz equation [28]. To make the calculus more accurate it is possible to measure the exact concentration of each component at the end of zero spatial frequency recording by additional methods. In any case, interesting information can be extracted solving the following equation for some particular cases:

$$
\frac{n_{p}^{2}-1}{n_{p}^{2}+2}=\frac{1}{\phi_{f}^{(p)}}\left[\frac{n_{f}^{2}-1}{n_{f}^{2}+2}-\sum \frac{n_{i}^{2}-1}{n_{i}^{2}+2} \phi_{f}^{(i)}\right],
$$

where $n_{f}$ is the final refractive index of the layer, $n_{p}$ is the refractive index of the polymer formed during exposition, $n_{i}$ is the refractive index of chemical compound $i$, and $\phi_{f}{ }^{(i)}$ is the final volume fraction for compound $i$. In the cases analyzed in this work, due to the high value of dye concentration, the samples show high absorption (the initial transmittances are around 1\%). In addition, at the end of exposing process we detected a weak presence of dye. Thus we assume that in the exposed zones all the components, except the binder (in the case of PVA/AA and PVA/NaAO) or liquid crystal (in PEA photopolymer), are polymerized. In this sense, radical polymerization models could be used to know the exact concentration of monomer and dye after illumination [3133]. With this assumption (5) can be reduced to:

$$
\frac{n_{p}^{2}-1}{n_{p}^{2}+2}=\frac{1}{\phi_{f}^{(p)}}\left[\frac{n_{f}^{2}-1}{n_{f}^{2}+2}-\frac{n_{C}^{2}-1}{n_{C}^{2}+2} \phi^{(C)}\right],
$$

where $n_{C}$ and $\phi^{(C)}$ are the refractive index and volume fraction of the constant component, binder in PVA materials, and liquid crystal in H-DPLC.

\section{Results and Discussion}

In this section we present the experimental data obtained for different material compositions and different thicknesses using both experimental setups for PVA photopolymers and only transmission analysis for H-DPLC.

4.1. PVA Photopolymers. In transmission analysis we can observe the effects of the refractive index changes and thickness variation during polymerization. The results for the new five compositions are presented in Figure 2. As can be seen the changes in the phase between exposed and nonexposed zones for compositions $\mathrm{A}$ and $\mathrm{E}$ are very weak (around $60^{\circ}$ ), therefore the refractive index variation and polymerization rate are small. It is important to note that the absorption of PRF is clearly lower than YE.

As the velocity of the reaction is very slow, after $1000 \mathrm{~s}$ the material continues changing in compositions A, E, and D (compositions without crosslinker). Compositions B and C (compositions with crosslinker) present very similar behavior in transmission analysis. In these compositions after $400 \mathrm{~s}$ the reaction stops and the layers are in saturation state (where the phase shift does not change with the illumination). Using composition $\mathrm{G}(\mathrm{AA}+\mathrm{BMA})$ the highest values of phase shift are obtained due to presence of the crosslinker.

In Figure 3, we have presented the results obtained in reflection analysis for NaAO-based compositions (A, B, C, $\mathrm{D}$, and $\mathrm{E})$. We can observe that the maximum shrinkage is around $3 \%$ for compositions $\mathrm{B}, \mathrm{C}$, and $\mathrm{D}$. This shrinkage is 


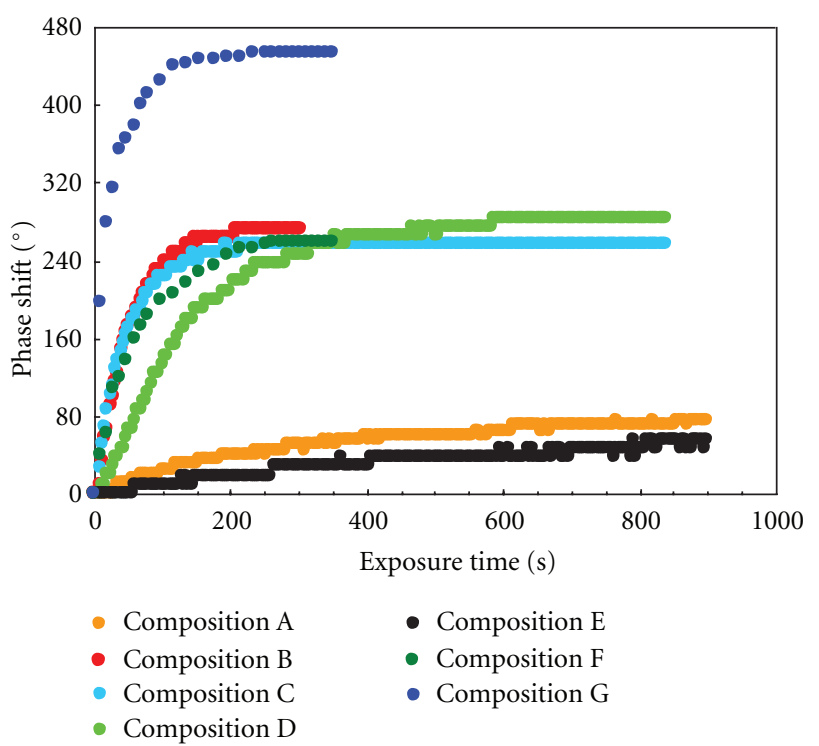

Figure 2: Phase shift as a function of the exposure time in transmission experiments for photopolymer compositions $\mathrm{A}, \mathrm{B}$, $\mathrm{C}, \mathrm{D}$, and E Biophotopol-derived compositions and F- and G(PVA/AA) based materials.

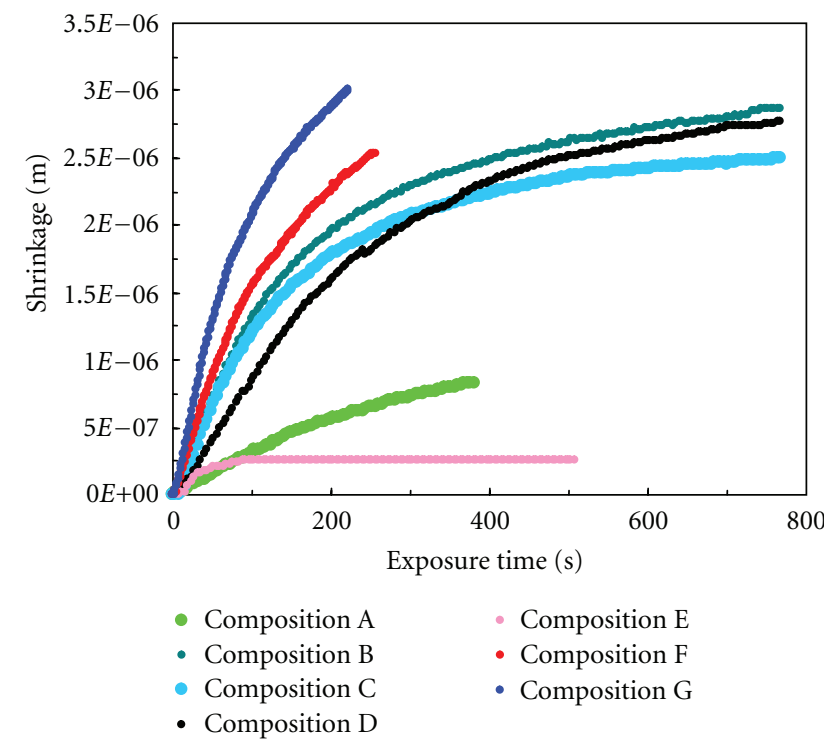

FIgURE 3: Shrinkages as a function of the exposure time for photopolymer compositions A, B, C, D, E, and F in reflection experiments.

large enough to store reflection diffractive elements using the relief variations [35]. On the other hand to use these materials as holographic memories the shrinkage should be reduced. As can be deduced from this and last figures compositions B, C, and D present similar behavior. The composition E, with PRF as a dye, the shrinkage is around $0.25 \mu \mathrm{m}$, nevertheless we cannot extract any significant information because the dye crystallizes during exposition.

In Figure 4 we depicted the results for some compositions obtained by the fitting based on (4). The results show us different values of $F_{R}$ for each composition and in Table 5.

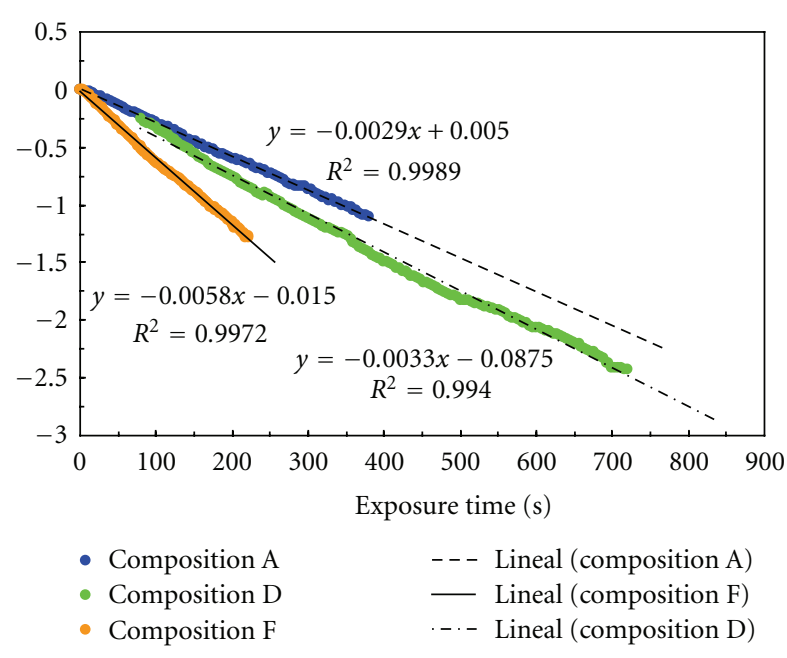

FIGURE 4: Fittings derived from (4) based on reflection experiments.

TABLE 5: Initial volume fractions and refractive indexes, $n$.

\begin{tabular}{lcccccc}
\hline Composition type & $\mathrm{A}$ & $\mathrm{B}$ & $\mathrm{C}$ & $\mathrm{D}$ & $\mathrm{F}$ & $\mathrm{G}$ \\
\hline$F_{R} \times 10^{-3}\left(\mathrm{~s}^{-1}\right)$ & 3.2 & 3.6 & 4.1 & 3.3 & 5.8 & 7.0 \\
$R^{2}$ & 0.999 & 0.993 & 0.992 & 0.994 & 0.997 & 0.994 \\
\hline
\end{tabular}

The correlation value of the fittings, $R^{2}$, indicates the good agreement between theory and experiments. In addition the compositions $\mathrm{A}$ and $\mathrm{D}$ present similar values of $F_{R}$ because the only variation is the monomer concentration. For composition $\mathrm{G}$ (AA+BMA) we measured the highest values of phase shift are obtained due to presence of the crosslinker. Maybe for holographic recording there are some small changes in $F_{R}$ values, because the experimental conditions present some differences such as the moving of monomers. Nevertheless we believe that using real zero spatial frequency a good estimation of the order of magnitude of $F_{R}$ can be obtain. The validity of these values has been confirmed in recent papers [35], where the values of zero spatial frequency were used to predict the formation of relief optical elements on the photopolymer surface.

To calculate the value of $\gamma$ in PVA/NaAO materials we exposed the material to two different exposure intensities 0.5 and $1 \mathrm{~mW} / \mathrm{cm}^{2}$ for composition type A and we calculated the polymerization rate value in each experiment using (4). The comparative result between the two exposures intensities are presented in Figure 5. With these data it is easy to obtain the value of $\gamma$. In our case the value obtained is 0.48 . This value is in good agreement with the value of 0.5 , usually assumed for photopolymers with low viscosity $[12,17]$. It is important to note that for low values of the parameter $\gamma$ the nonlinearity is increased.

It is obvious that the phase shift obtained depends directly of the photopolymer thickness. In [15] this method was applied to characterize layers thicker than $0.5 \mathrm{~mm}$ able to store high quantity of data pages [5]. Results provided by (6) are presented in Table 6 (we have omitted composition G due to its different behavior). These values for $n_{p}$ are important when they are compared with the monomers ones; that is, 


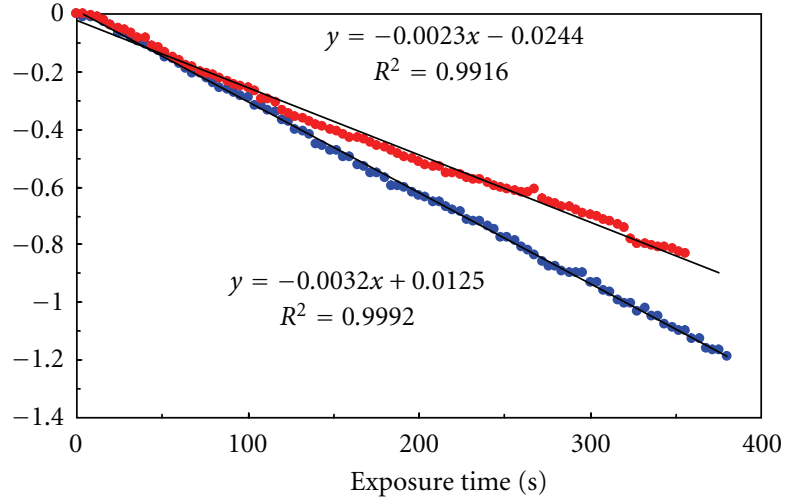

- $1 \mathrm{~mW} / \mathrm{cm}^{2} \quad$ Lineal $\left(1 \mathrm{~mW} / \mathrm{cm}^{2}\right)$

- $0.5 \mathrm{~mW} / \mathrm{cm}^{2} \quad$ Lineal $\left(0.5 \mathrm{~mW} / \mathrm{cm}^{2}\right)$

Figure 5: Fittings derived from (4) based for different exposure intensities $\left(0.5 \mathrm{~mW} / \mathrm{cm}^{2}\right.$ and $\left.1 \mathrm{~mW} / \mathrm{cm}^{2}\right)$.

TABLE 6: Fitted polymer refractive index.

\begin{tabular}{lccccc}
\hline Composition type & $\mathrm{A}$ & $\mathrm{B}$ & $\mathrm{C}$ & $\mathrm{D}$ & $\mathrm{E}$ \\
\hline$n_{p}$ & 1.578 & 1.603 & 1.585 & 1.595 & 1.583 \\
\hline
\end{tabular}

the refractive index of acrylamide is clearly lower than the $\mathrm{NaAO}$ refractive index [17]. To determinate these values we have followed the steps indicated in [15-17]. Furthermore we want to remark that the main important point is to compare $n_{m}$ with $n_{p}$ and these parameters with the value of the refractive index of the binder, $n_{b}(5)$. In this sense the importance of crosslinker to increase $n_{p}$ has been demonstrated using the zero frequency technique. To obtain a better analysis of the data presented in Table 6 it is important to note that the refractive index of $\mathrm{NaOA}$ is 1.565 [17].

4.2. H-DPLC Photopolymers. In order to obtain information about the effect of incorporating CL in the photopolymerizable solution using real zero frequency recording, the phase shift between illuminated and nonilluminated zones for compositions C1, C2, C3, C4, and C5 is shown in Figure 6. As can be seen in this figure, the presence of CL molecules produces a decrease in phase shift. The values of $n_{p}$ obtained for C5, C4, C3, and C5 (without LC) using (6) are 1.492, $1.492,1.495,1.496$, and 1.496, respectively. Therefore we can conclude that incorporation of LC molecules results in only a small change in polymer refractive index. As zero spatial frequency recording prevents diffusion of liquid crystalline molecules into dark regions and of monomers to bright regions, the uniform concentration of LC throughout the whole layer makes it difficult for the refractive index to change.

Following the steps described previously and using (4), it is possible to obtain the polymerization rate for each composition. The values obtained are represented in Table 7. As can be seen in this figure the correlation parameter indicates that there is agreement between experimental data and theoretical behaviour. From the analysis of Table 7 the high values of the polymerization rates obtained can be deduced. These values

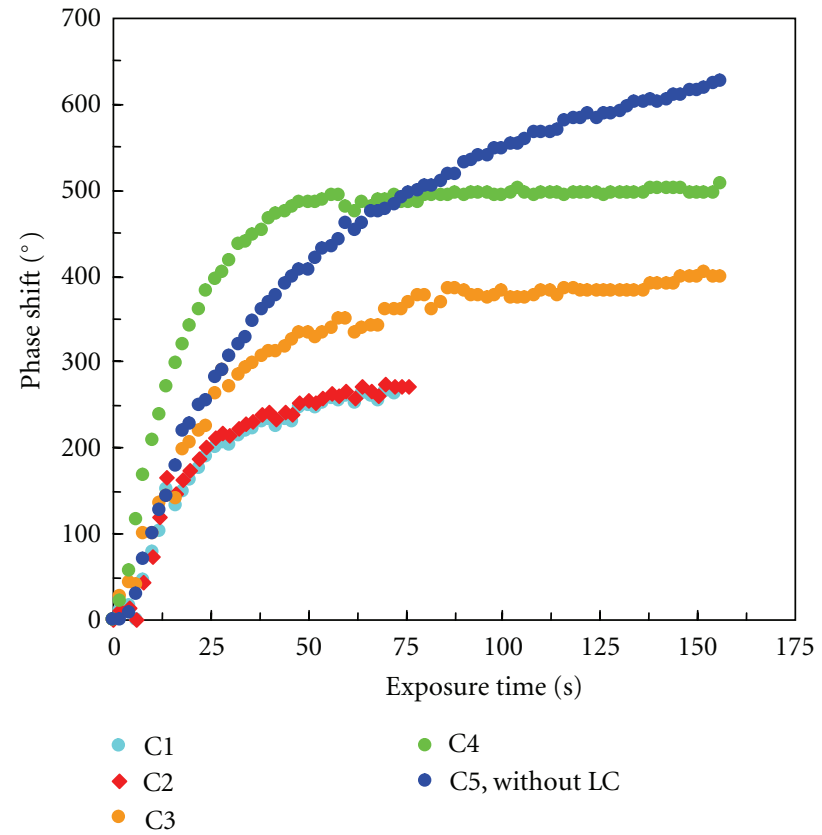

Figure 6: Behavior of photopolymers C1, C2, C3, C4, and C5 at the zero spatial frequency limit in transmission.

TABLE 7: Initial volume fractions and refractive indexes, $n$.

\begin{tabular}{lccccc}
\hline Composition type & C1 & C2 & C3 & C4 & C5 \\
\hline$F_{R} \times 10^{-2}\left(\mathrm{~s}^{-1}\right)$ & 4.38 & 4.86 & 3.56 & 5.50 & 4.54 \\
$R^{2}$ & 0.984 & 0.986 & 0.977 & 0.997 & 0.994 \\
\hline
\end{tabular}

are 10 times higher than the ones obtained for PVA materials analyzed in Table 5, due to the multifunctional monomer used in H-PDLC materials. To fit accurately the polymerization rate in these materials the temperature is a fundamental parameter. In general, in Table 7 we can detect a decrease in the polymerization when high values of LC concentration are included in the composition. The exception is the C3 with very low polymerization rate and correlation parameter. As can be seen the curve grows very quickly from 0 to $50 \mathrm{~s}$ and then the phase shifts increases slowly, as if the residual monomer polymerizes with difficulties, maybe produced by Trommsdorff effect [36] and the cooling of the layer.

\section{Conclusions}

Using zero frequency recording we characterized different types of photopolymers with and without liquid crystal molecules, cover plating, crosslinker, and so forth. Using this method we were able to analyze polymerization separately from the diffusion and thus discuss the influence of the different components such as liquid crystal molecules, crosslinker, and so forth on grating formation. We also presented some simply models using interferometric methods to fit the polymerization rate, the refractive index of polymers and the relation between exposure intensity on polymerization. We 
have shown how this method can be used to obtain photopolymer layers with desired properties. Finally we think that this method can be applied to fit some of the multitude of parameters that take place in the light absorption in photopolymers materials. All these parameters should be taking into account in order to design holographic diffusion model to predict the behavior of these photopolymers. In our opinion the viability of these three types of photopolymers for diffractive and holographic application has been demonstrated.

\section{Acknowledgments}

This work was supported by the Ministerio de Ciencia e Innovación of Spain under Projects FIS2011-29803-C02-01 and FIS2011-29803-C02-02 and by the Generalitat Valenciana of Spain under Project PROMETEO/2011/021.

\section{References}

[1] J. Neumann, K. S. Wieking, and D. Kip, "Direct laser writing of surface reliefs in dry, self-developing photopolymer films," Applied Optics, vol. 38, no. 25, pp. 5418-5421, 1999.

[2] X. T. Li, A. Natansohn, and P. Rochon, "Photoinduced liquid crystal alignment based on a surface relief grating in an assembled cell," Applied Physics Letters, vol. 74, no. 25, pp. 37913793, 1999.

[3] A. Márquez, S. Gallego, M. Ortuño et al., "Generation of diffractive optical elements onto a photopolymer using a liquid crystal display," in Optical Modelling and Design, vol. $7717,77170 \mathrm{D}$ of Proceedings of the SPIE, Brussels, Belgium, April 2010.

[4] M. D. Lechner, "Photopolymers for optical memories and waveguides," Electronic Properties of Polymers and Related Compounds, vol. 63, pp. 301-308, 1985.

[5] H. J. Coufal, D. Psaltis, and G. T. Sincerbox, Holographic Data Storage, Springer Series in Optical Sciences, Springer, Berlin, Germany, 2000.

[6] A. Márquez, C. Neipp, A. Beléndez, S. Gallego, M. Ortuño, and I. Pascual, "Edge-enhanced imaging with polyvinyl alcohol/acrylamide photopolymer gratings," Optics Letters, vol. 28, no. 17, pp. 1510-1512, 2003.

[7] G. Manivannan and R. A. Lessard, "Trends in holographic recording materials," Trends in Polymer Science, vol. 2, pp. 282290, 1994.

[8] K. Curtis, L. Dhar, L. Murphy, and A. Hill, Future Developments, in Holographic Data Storage: From Theory to Practical Systems, John Wiley \& Sons, 2010.

[9] J. T. Sheridan and J. R. Lawrence, "Nonlocal-response diffusion model of holographic recording in photopolymer," Journal of the Optical Society of America A, vol. 17, no. 6, pp. 1108$1114,2000$.

[10] M. R. Gleeson, S. Liu, and J. T. Sheridan, "The production of primary radicals in photopolymers during holographic exposure," Optik, vol. 121, no. 24, pp. 2273-2275, 2010.

[11] I. Naydenova, E. Mihaylova, S. Martin, and V. Toal, "Holographic patterning of acrylamide-based photopolymer surface," Optics Express, vol. 13, no. 13, pp. 4878-4889, 2005.

[12] G. Zhao and P. Moroulis, "Extension of diffusion model of holograhic photopolymer," Optics Communications, vol. 15, pp. 528-532, 1995.
[13] J. V. Kelly, M. R. Gleeson, C. E. Close et al., “Temporal analysis of grating formation in photopolymer using the nonlocal polymerization-driven diffusion model," Optics Express, vol. 13, no. 18, pp. 6990-7004, 2005.

[14] S. Gallego, A. Márquez, D. Méndez, S. Marini, A. Beléndez, and I. Pascual, "Spatial-phase-modulation-based study of polyvinyl-alcohol/acrylamide photopolymers in the low spatial frequency range," Applied Optics, vol. 48, no. 22, pp. 44034413, 2009.

[15] S. Gallego, A. Márquez, D. Méndez et al., "Real-time interferometric characterization of a polyvinyl alcohol based photopolymer at the zero spatial frequency limit," Applied Optics, vol. 46, no. 30, pp. 7506-7512, 2007.

[16] S. Gallego, A. Márquez, D. Méndez et al., "Analysis of PVA/AA based photopolymers at the zero spatial frequency limit using interferometric methods," Applied Optics, vol. 47, no. 14, pp. 2557-2563, 2008.

[17] S. Gallego, A. Márquez, M. Ortuño, S. Marini, and J. Francés, "High environmental compatibility photopolymers compared to PVA/AA based materials at zero spatial frequency limit," Optical Materials, vol. 33, no. 3, pp. 531-537, 2011.

[18] A.J. Bergeron, F. Gauvin, D. Gagnon, H. Gingras, H. H. Arsenault, and M. Doucet, "Phase calibration and applications of a liquid crystal spatial light modulator," Applied Optics, vol. 34, pp. 5133-5139, 1995.

[19] S. Calixto, "Dry polymer for holographic recording," Applied Optics, vol. 26, pp. 3904-3909, 1987.

[20] S. Blaya, R. Mallavia, L. Carretero, A. Fimia, and R. F. Madrigal, "Highly sensitive photopolymerizable dry film for use in real time holography," Applied Physics Letters, vol. 73, no. 12, pp. 1628-1630, 1998.

[21] S. Martin, P. E. Leclere, Y. L. M. Renotte, V. Toal, and Y. F. Lion, "Characterization of an acrylamide-based dry photopolymer holographic recording material," Optical Engineering, vol. 33, p. 3942, 1994.

[22] M. Ortuño, S. Gallego, C. García, C. Neipp, A. Beléndez, and I. Pascual, "Optimization of a $1 \mathrm{~mm}$ thick PVA/acrylamide recording material to obtain holographic memories: method of preparation and holographic properties," Applied Physics B, vol. 76, no. 8, pp. 851-857, 2003.

[23] F. T. O’Neill, A. J. Carr, S. M. Daniels et al., "Refractive elements produced in photopolymer layers," Journal of Materials Science, vol. 40, no. 15, pp. 4129-4132, 2005.

[24] M. Friedman, "Chemistry, biochemistry, and safety of acrylamide. A review," Journal of Agricultural and Food Chemistry, vol. 51, no. 16, pp. 4504-4526, 2003.

[25] M. Ortuño, E. Fernández, S. Gallego, A. Beléndez, and I. Pascual, "New photopolymer holographic recording material with sustainable design," Optics Express, vol. 15, no. 19, pp. 12425-12435, 2007.

[26] M. R. Gleeson, J. V. Kelly, C. E. Close, F. T. O’Neill, and J. T. Sheridan, "Effects of absorption and inhibition during grating formation in photopolymer materials," Journal of the Optical Society of America B, vol. 23, no. 10, pp. 2079-2088, 2006.

[27] S. Gallego, Modelización del comportamiento holográfico de un fotopolimero de polivinilalcohol/acrilamida [Ph.D. thesis], Univertity of Alicante, 2005.

[28] M. Born and E. Wolf, Principles of Optics, Pergamon Press, Oxford, UK, 1980.

[29] S. Gallego, C. Neipp, M. Ortuño, A. Beléndez, E. Fernández, and I. Pascual, "Analysis of monomer diffusion in depth in photopolymer materials," Optics Communications, vol. 274, no. 1, pp. 43-49, 2007. 
[30] S. Gallego, M. Ortuño, C. Neipp et al., “3-dimensional characterization of thick grating formation in PVA/AA based photopolymer," Optics Express, vol. 14, no. 12, pp. 5121-5128, 2006.

[31] S. Liu, M. R. Gleeson, J. Guo, and J. T. Sheridan, "Optical characterization of photopolymers materials: theoretical and experimental examination of primary radical generation," Applied Physics B, vol. 100, pp. 559-569, 2010.

[32] D. Sabol, M. R. Gleeson, S. Liu, and J. T. Sheridan, "Photoinitiation study of Irgacure 784 in an epoxy resin photopolymer," Journal of Applied Physics, vol. 107, no. 5, Article ID 053113, 2010.

[33] S. Liu, M. R. Gleeson, J. Guo, and J. T. Sheridan, "High intensity response of photopolymer materials for holographic grating formation," Macromolecules, vol. 43, no. 22, pp. 94629472, 2010.

[34] S. Gallego, A. Márquez, M. Ortuño et al., "Surface relief model for photopolymers without cover plating," Optics Express, vol. 19, no. 11, pp. 10896-10906, 2011.

[35] S. Gallego, A. Márquez, M. Ortuño, J. Francés, I. Pascual, and A. Beléndez, "Relief diffracted elements recorded on absorbent photopolymers," Optics Express, vol. 20, pp. 11218-11231, 2012.

[36] J. V. Kelly, F. T. O’Neill, J. T. Sheridan, C. Neipp, S. Gallego, and M. Ortuno, "Holographic photopolymer materials: nonlocal polymerization-driven diffusion under nonideal kinetic conditions," Journal of the Optical Society of America B, vol. 22, no. 2, pp. 407-416, 2005. 

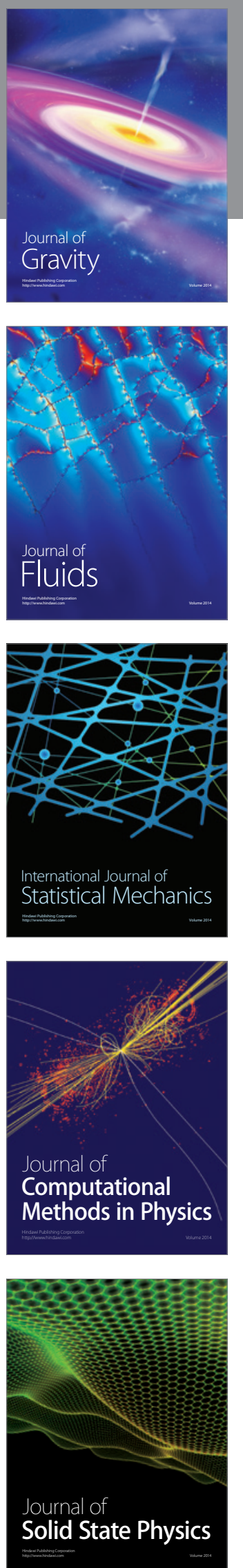

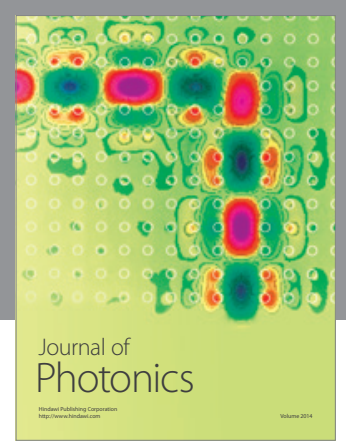

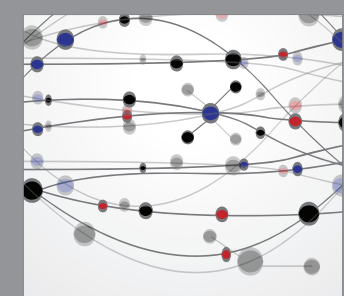

The Scientific World Journal
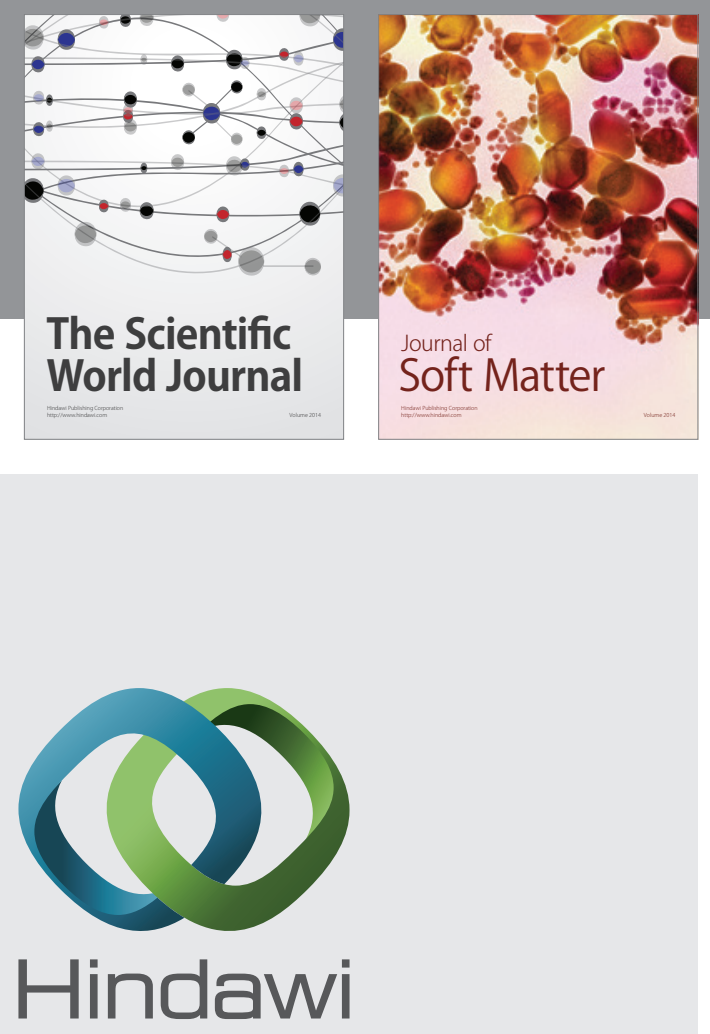

Submit your manuscripts at

http://www.hindawi.com
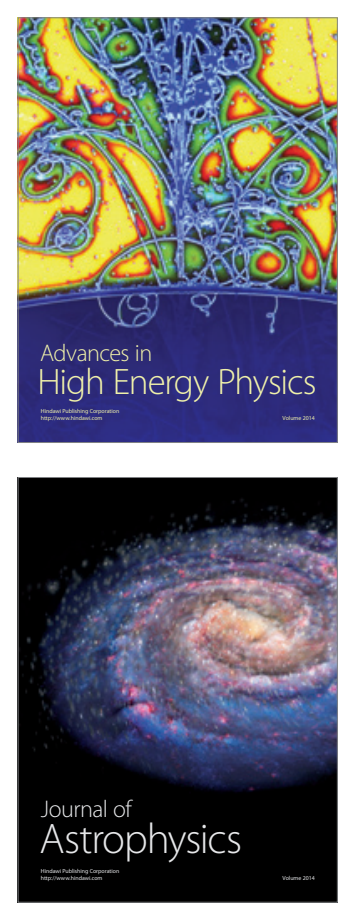
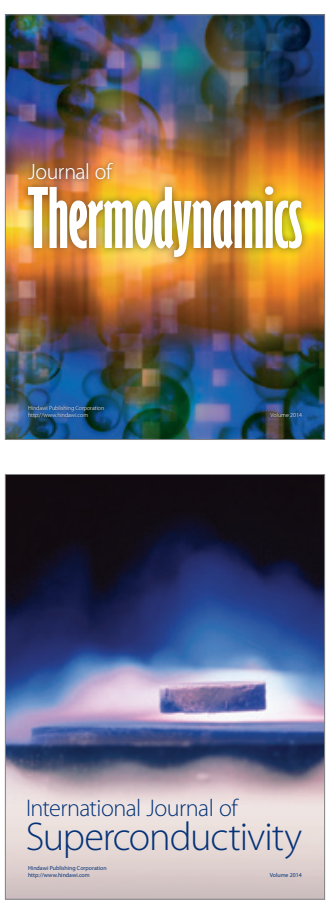
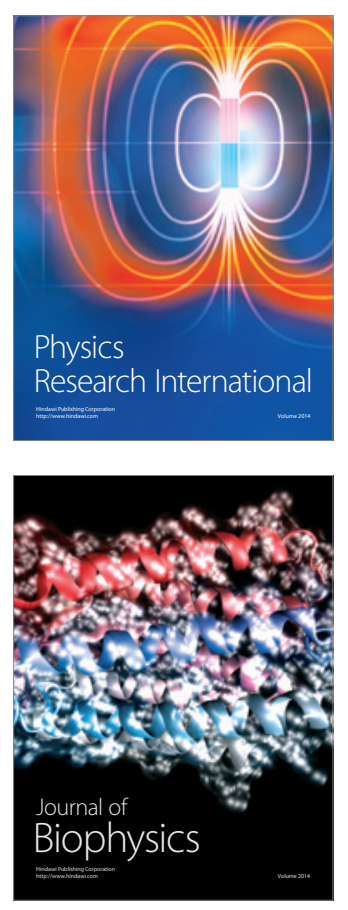
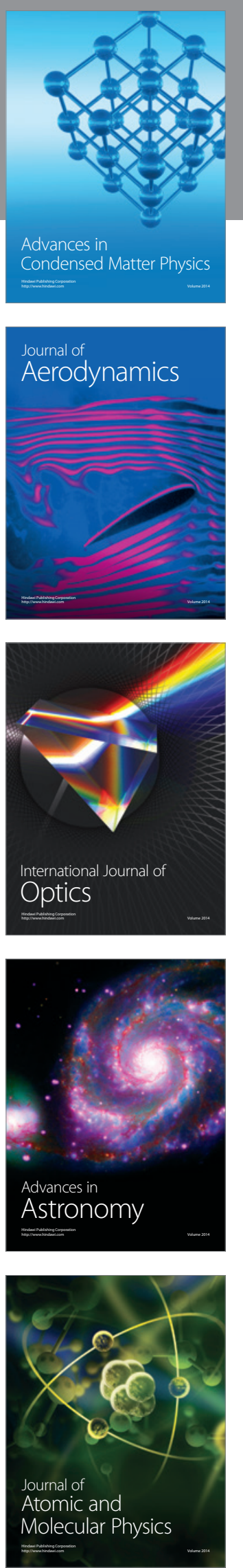